\title{
LAS UNIVERSIDADES Y SUS PRÁCTICAS DE VINCULACIÓN CON LA SOCIEDAD
}

\author{
IvÁN RuEDA ${ }^{1}$ \\ BYron Acosta ${ }^{2}$ \\ FABián Cueva ${ }^{3}$
}

\begin{abstract}
RESUMEN: El objetivo de esta investigación es determinar si las prácticas de vinculación realizadas por las universidades son realmente de vinculación con la sociedad. Se analizaron los reportes de actividades de vinculación de 60 universidades, se contrastó lo reportado y se validó si las actividades ejecutadas son realmente de vinculación y de qué tipo son. Los resultados muestran que la acción social y la educación continua son las actividades de vinculación que las universidades realizan en su mayoría. Además, la transferencia de tecnología y creación de empresas son las actividades de vinculación menos desarrolladas. Finalmente, el $25 \%$ de las universidades clasifica al seguimiento a graduados como práctica de vinculación con la sociedad, aunque dicha actividad no es considerada de vinculación.
\end{abstract}

Palabras-clave: Vinculación con la sociedad. Transferencia de tecnología. Universidades.

\section{UNIVERSITIES AND THEIR PRACTICES OF OUTREARCH WITH SOCIETY}

\begin{abstract}
The objective of this research is to determine if the practices of outrearch carried out by the universities are really of outrearch. The reports of outrearch activities of 60 universities were analyzed. The information reported by each institution was checked and validated based on the literature to see if the activities developed are really related and of what type they are. The results show that social action and continuing education are the types of outrearch activities that most universities carry out. On the other hand, the transfer of technology and the creation of companies are the activities of outrearch less development. In addition, $25 \%$ of the universities classify the follow-up to graduates as practice of outrearch, although such activity is not considered as of outrearch.
\end{abstract}

Keywords: Outrearch. Technology transference. Universities.

1.Pontificia Universidad Católica del Ecuador, Facultad de Ciencias Administrativas - Quito (Pichincha), Ecuador. E-mail: iarueda@puce.edu.ce 2.Escuela Politécnica Nacional, Facultad de Ciencias Administrativas - Quito (Pichincha), Ecuador. E-mail: byron.acosta@epn.edu.ec 3.Pontificia Universidad Católica del Ecuador, Facultad de Ciencias Administrativas - Quito (Pichincha), Ecuador. E-mail: efcuevab@puce.edu.ec Producto del proyecto: Apropiación de las IES de las actividades de vinculación con la sociedad: ¿Cómo las Universidades ecuatorianas entienden y aplican el concepto de vinculación? 


\title{
AS UNIVERSIDADES E SUAS PRÁTICAS DE VINCULAÇÃO COM A SOCIEDADE
}

\begin{abstract}
RESUMO: O objetivo desta pesquisa é determinar se as práticas de vinculação realizadas pelas universidades são realmente vinculadas à sociedade. Foram analisados os relatórios de atividades de vinculação de 60 universidades para comparar e verificar se as atividades executadas foram realmente de vinculação e do que tipo elas são. Os resultados mostram que a ação social e a educação continuada são as práticas de vinculação mais executadas pelas universidades. Além disso, a transferência de tecnologia y a criação de empresa são as atividades que em menor grau se executam. Finalmente, 25\% das universidades classificam o acompanhamento de graduação como prática de vinculação, embora essa atividade não seja considerada como de vinculação.
\end{abstract}

Palavras-chave: Vinculação com a sociedade. Transferência de tecnologia. Universidades

\section{Introducción}

$\mathrm{L}$ as universidades son instituciones sociales cuya misión debe ser cumplida por medio de tres procesos fundamentales: la formación de profesionales e investigadores; la generación y difusión de conocimiento; y la vinculación con la sociedad. Estos elementos están presentes desde el origen de las universidades y su forma de cumplirlas ha variado a lo largo del tiempo. La universidad es una institución para toda la sociedad; moldea y contribuye al establecimiento de dinámicas sociales, y constituye una referencia educativa para estudiantes, profesores y la sociedad (PALEARI; DONINA; MEOLI, 2015). Para estas instituciones, el crecimiento debe referirse a la calidad de sus servicios y sus vínculos con la sociedad, las empresas y el mundo exterior; de hecho, tanto la formación profesional como la académica deben estar más abiertamente disponibles y más profundamente conectados con los entornos empresariales e institucionales (PALEARI; DONINA; MEOLI, 2015).

Las nuevas necesidades y demandas de la sociedad exigen que las universidades cambien su enfoque y no solo se encarguen de la formación de profesionales. Entre las presiones que la universidad enfrenta de la sociedad están la creciente demanda por educación superior, la reducción de recursos públicos, y la necesidad por flexibilidad y capacidad de respuesta a las necesidades económicas y sociales (PALEARI; DONINA; MEOLI, 2015). Precisamente, cada vez más la sociedad demanda por parte de la universidad mayor participación en los acontecimientos sociales (MARTÍNEZ et al., 2012). Para Audretsch (2014), el papel de la universidad en la sociedad ha evolucionado con el tiempo, principalmente debido a las presiones por el crecimiento y desempeño económico. Sin embargo, el objetivo de la universidad en una sociedad emprendedora, no es solo el promover la transferencia de tecnología e incrementar el número de empresas emergentes, sino asegurar que las personas prosperen en la sociedad (AUDRETSCH, 2014). Para esto, las universidades deben transformarse para responder a los cambios en las demandas y necesidades de la sociedad y sus stakeholders (PALEARI; DONINA; MEOLI, 2015).

Los gobiernos alientan a las universidades a asumir una mayor responsabilidad por el desarrollo económico, transformando el conocimiento que generan en productos y servicios para el mercado; por otro lado, se les encarga también trabajar con las comunidades para aliviar los excesos sociales y económicos del mercado (BOURKE, 2013). El que las universidades trabajen con una visión de tipo económica no las desvirtúa 
de su esencia como institución, este enfoque económico debe ser equilibrado con el enfoque social. Lo que aleja a la universidad de su misión es trabajar con un único enfoque; es decir, tanto la perspectiva económica como la social deben estar relacionadas y aplicadas en las acciones que desarrollan estas instituciones; especialmente en el campo de la investigación. No aprovechar comercialmente en ningún momento el conocimiento generado por las universidades es un error; lo incorrecto es solo hacer eso, dejando de lado la responsabilidad que tienen con la sociedad.

Según Bourke (2013), existe presión para que las universidades produzcan evidencias tangibles que demuestren cómo están promoviendo investigaciones socialmente receptivas y cuál ha sido su contribución para las comunidades locales; no obstante, poco se ha hecho para situar dichas transformaciones como evidencia de cambios en las relaciones sociales y del compromiso de la investigación que realiza la universidad con la sociedad civil.

Las universidades contemporáneas tienen un papel central en la conexión entre educación, investigación e innovación (PALEARI; DONINA; MEOLI, 2015); en este nuevo contexto han surgido nuevos roles y tareas, que se espera que ellas realicen. Estas nuevas actividades se centran en la relación universidad-sociedad, y en cómo la primera disemina y aplica el conocimiento generado de forma comercial o no-comercial. Por lo tanto, la universidad debe ser vista como piedra angular del renovado concepto de sociedad, cuestionándose sobre qué rol la educación superior y la investigación jugarán en la construcción de la nueva sociedad (PALEARI; DONINA; MEOLI, 2015). La utilización de los resultados de la investigación y el conocimiento generado en las universidades son un importante recurso para reforzar el crecimiento económico en la sociedad; esta aplicación debe suceder no solo mediante la concesión de licencias de las patentes de las universidades o por la creación de nuevas empresas, sino también por compromisos académicos en diferentes procesos interactivos como conferencias, colaboraciones y publicaciones (JONSSON; BARALDI; LARSSON, 2015). En ese sentido, el primer paso, es interiorizar el impacto económico y social como objetivos finales en las universidades, sin eso, las actividades de vinculación corren el riesgo de ser vistas como simples oportunidades generadoras de ingresos (HATAKENAKA, 2005).

De esta manera, las universidades se tornan fuentes importantes de conocimiento, tecnología y capital humano calificado que proveen de ideas valiosas, apoyan a las nuevas industrias, se comprometen con la innovación y con el desarrollo de emprendimientos dinámicos apalancadas por su tercera misión vinculación con la sociedad (RANGA et al., 2016). Si los vínculos entre enseñanza, investigación y la tercera misión no están lo suficientemente desarrollados, surgen desafíos para la universidad en términos financieros, de calidad y de relevancia (RANGA et al., 2016). Según Ranga et al. (2016), la mayoría de las universidades carecen de elementos que faciliten la colaboración universidad-industria, la transferencia de tecnología y la provisión de servicios empresariales y sociales.

Las universidades deben cumplir su misión con base en tres componentes: la educación, la investigación y la vinculación con la sociedad; estos componentes se relacionan entre si, creando y manteniendo una comunicación bidireccional entre ellos (SOLEIMANI; TABRIZ; SHAVARINI, 2016). La educación debe basarse en investigación y la investigación debe formarse en función de las necesidades de la sociedad (SOLEIMANI; TABRIZ; SHAVARINI, 2016). No obstante, una critica común a la universidad es que ella se ha apartado del mundo real y realiza la investigación en laboratorios cerrados, olvidándose de las necesidades reales de la sociedad (SOLEIMANI; TABRIZ; SHAVARINI, 2016). Debido a esto, las universidades precisan moverse hacia una dirección que les permita apoyar su nuevo rol de participación en el desarrollo económico (SOLEIMANI; TABRIZ; SHAVARINI, 2016).

Entender y analizar el papel de la universidad en el contexto de hoy, es uno de los tópicos importantes en la investigación en ciencias sociales. Por ejemplo, Paleari, Donina y Meoli (2015) cuestionan cuál es el 
papel que debe tener la universidad en la sociedad del siglo XXI, explicando que este nuevo rol debe estar estrictamente relacionado a la idea de sociedad en la que queremos vivir. Con base en este contexto el objetivo de esta investigación es determinar si las prácticas de vinculación realizadas por las universidades son realmente de vinculación con la sociedad.

\section{Revisión de la literatura}

\section{La relación entre Vinculación con la Sociedad y Transferencia de Tecnología}

Las universidades además de generar conocimiento son responsables de transferirlo a la sociedad. Para Audretsch (2014), la simple generación de conocimiento no asegura que éste pueda ser diseminado para impulsar la actividad innovadora y el crecimiento económico. Precisamente, el conocimiento se transforma en un importante motor para el desarrollo económico, solo cuando las universidades lo difunden y los skateholders lo aplican (AUDRETSCH, 2014). Para Necochea-Mondragón, Pineda-Domínguez y Soto-Flores (2013) las universidades, desde finales del Siglo XX, comenzaron a ser consideradas como proveedoras de conocimiento útil para ser explotado en términos prácticos por la industria. Desde esta prespectiva, los avances científicos obtenidos a lo largo del siglo XX llevaron a la universidad a tratar de forma más objetiva su rol de investigadora, orientando parte de sus actividades a la atención de las demandas económicas (BEZERRA, 2012). Cuando la universidad lleva hacia la sociedad el conocimiento que desarrolla, está realizando procesos de transferencia de tecnología, con el propósito de solucionar o satisfacer las necesidades de la colectividad. De una manera simple, el término tansferencia de tecnología se define como el proceso de movimiento de tecnología de una entidad a otra, teniendo en cuenta una condición básica, que la transferencia es exitosa si la entidad receptora puede utilizar efectivamente la tecnología para obtener beneficios empresariales (NECOECHEA-MONDRAGÓN; PINEDA-DOMÍNGUEZ; SOTO-FLORES, 2013). Es decir, la vinculación con la sociedad es el resultado de la transferencia de tecnología que realiza la universidad; dicho de otra forma, en el momento que la universidad realiza transferencia de conocimientos simultáneamente está realizando vinculación con la sociedad.

La transferencia de tecnología (TT) entre la universidad y la industria es una forma de cooperación y, por lo tanto, tiene diferentes objetivos particulares para cooperar entre sí (FENG; CHEN; WANG, 2012). Las universidades además de difundir conocimiento, ayudan a las empresas a desarrollar aplicaciones prácticas; concibiendo a las universidades como un colaborador para la economía basada en el conocimiento (FENG; CHEN; WANG, 2012). Desde el punto de vista del conocimiento, la TT es un proceso que propaga o facilita la adquisición de un conjunto de conocimientos, experiencias e información de una organización a otra (FENG; CHEN; WANG, 2012). En ese sentido, los programas de TT son importantes para las tres misiones sustantivas de las universidades -enseñanza, investigación y vinculación-, por proporcionar: un mecanismo para que los resultados relevantes de la investigación sean transferidos a la sociedad; un asesoramiento para los investigadores en el manejo de la relación comercial con la industria; un método para facilitar y fomentar la investigación industrial; una fuente de fondos no restringidos para investigación adicional; y una fuente de experiencia en licenciamiento y negociación de contratos industriales (FENG; CHEN; WANG, 2012).

Como lo manifiesta Martínez y Soto (2007), para que este proceso se dé se puede empezar por la prestación de servicios especializados y programas de capacitación. Consecutivamente se pueden celebrar contratos de consultoría y asistencia técnica en áreas donde la universidad tenga un alto nivel de competencia. Para, posteriormente, pasar a las fases superiores relacionadas con la investigación.

La Fig. 1 presenta el proceso de transferencia de tecnología en universidades. Para que la institución pueda desarrollar procesos exitosos de TT, debe contar con la suficiente investigación y un sólido grupo de 
investigadores, con un nivel competitivo internacional; ya que el insumo principal para que exista TT son los resultados de I+D generados por sus investigadores (MARTÍNEZ; SOTO, 2007). Según Feng, Chen y Wang (2012), antes de realizar TT las universidades deben lograr abundantes resultados de investigación, y contar con recurso humano capacitado. Además, deben mantener una buena relación con la industria y los stakeholders; si estás condiciones se cumplen, la universidad tendrá los suficientes recursos para transferir tecnología. A esto se suma la pertinencia social de la universidad, donde los resultados de la investigación, docencia y vinculación con la sociedad puedan ser transferidos como paquetes tecnológicos, generando un alto impacto en el entorno (MARTÍNEZ et al., 2012). La integración de las funciones de docencia, investigación y vinculación, permiten que los procesos de transferencia de resultados de investigación (servicios, laboratorios, consultoría, licenciamiento de patentes y registros, capacitación), se conviertan en oferta o portafolio de servicios tecnológicos (MARTíNEZ et al., 2012).

Sobre esta perspectiva, sin investigación es imposible hacer TT y sin TT no se puede realizar vinculación con la sociedad; es decir, la TT viabiliza para que las universidades consoliden su integración

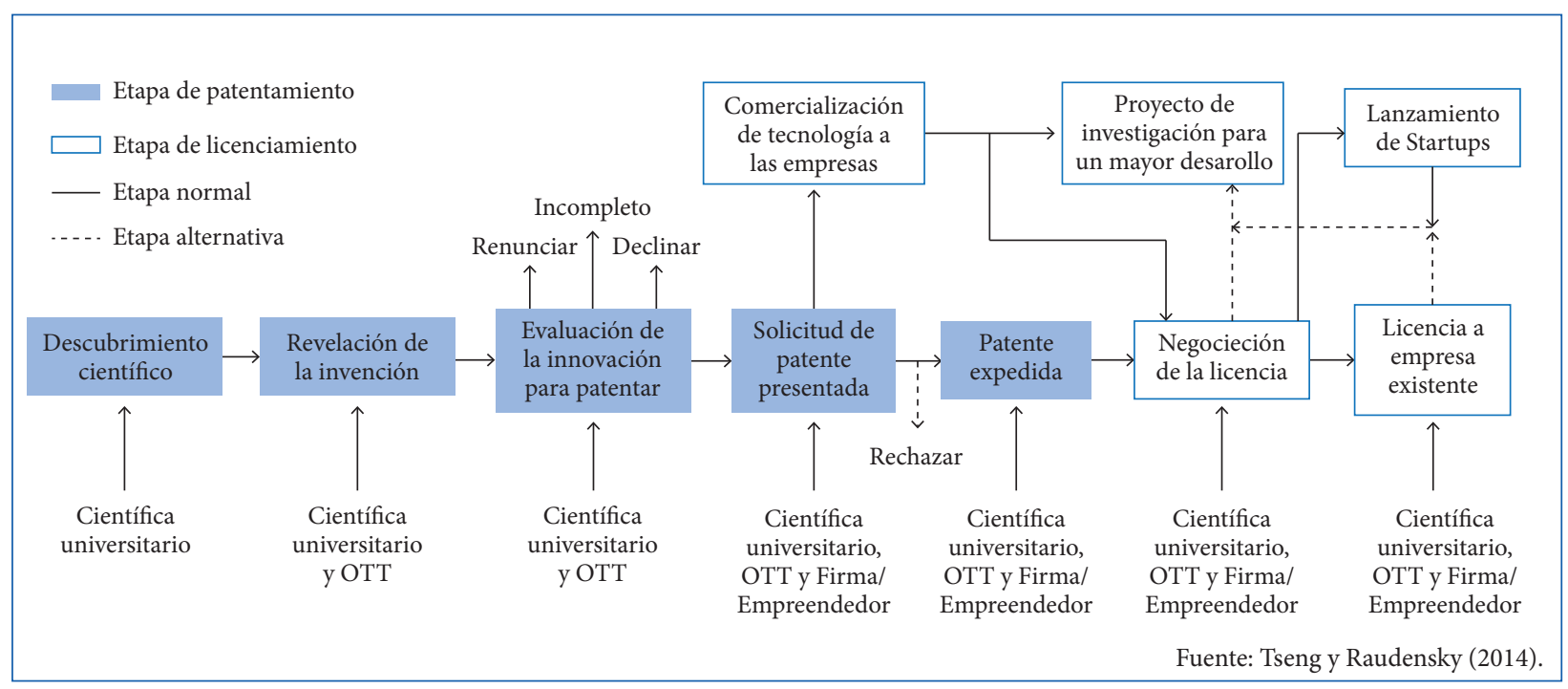

Figura 1. Proceso de TT en universidades.

con la sociedad de forma fidedigna. Por lo tanto, las universidades deben entender que el conocimimiento que ellas crean y difunden debe estar vinculado a satisfacer las necesidades sociales, económicas y políticas, creando soluciones a largo plazo. Como lo especifica Alessandrini, Klose y Pepper (2013), la TT es algo más que la simple comercialización del $\mathrm{I}+\mathrm{D}$, se trata de sacar descubrimientos y resultados de investigación de manera más general y encontrar aplicaciones que sean útiles para la humanidad. En esencia, la vinculación con la sociedad es la ejecución de actividades de tranferencia de tecnología; sin embargo, para que se realice TT primero debe existir I+D desarrollada por la universidad. Por lo tanto, una condicion fundamental para realizar vinculación con la sociedad es que existan resultados de investigación que puedan ser transferibles.

Como consecuencia, la TT tiene tanto objetivos sociales como comerciales; sin embargo, para que dichos objetivos puedan ser alcanzados, las universidades necesitan ser más emprendedoras. Precisamente, la TT es una oportunidad para viabilizar la vinculación de la universidad con el entorno social, ofreciendo servicios espacializados y programas de capacitación; para posteriormente ofrecer contratos de consultoría y asistencia técnica en las áreas donde la institucción tenga un alto nivel de competencia (MARTíNEZ et al., 2012). Como lo explica Audretsch (2014), las universidades necesitan involucrarse más proactivamente en actividades empresariales para facilitar el desbordamiento de conocimiento para difundir o comercializar sus 
resultados fuera de su hábitat. Los mecanismos de transferencia de tecnología se pueden concentrar en tres grupos. El primero, relacionado con la gestión de la propiedad intelectual, como la solicitud y la obtención de patentes basadas en los resultados de la investigación, comercialización de la propiedad intelectual e ingresos provenientes de acuerdos de licenciamiento (ALESSANDRINI; KLOSE; PEPPER, 2013; FENG; CHEN; WANG, 2012; GONZÁLEZ-PERNÍA; KUECHLE; PEÑA-LEGAZKUE, 2013; NECOECHEAMONDRAGÓN; PINEDA-DOMÍNGUEZ; SOTO-FLORES, 2013; RANGA et al., 2016; SCHOEN; POTTERIE; HENKEL, 2014; VINIG; LIPS, 2015). El segundo grupo relacionado con servicios de consultoría, prestación de servicios especializados, programas de capacitación y educación continua, asistencia técnica; gestión de contratos de investigación; servicios de planificación de negocios para inventores e investigadores; y acuerdos de investigación y desarrollo coorporativo (ALESSANDRINI; KLOSE; PEPPER, 2013; MARTÍNEZ et al., 2012; NECOECHEA-MONDRAGÓN; PINEDA-DOMÍNGUEZ; SOTO-FLORES, 2013; RANGA et al., 2016). Y el tercer grupo relacionado con servicios de spin-out (SCHOEN; POTTERIE; HENKEL, 2014), creación de spin-offs (GONZÁLEZ-PERNÍA; KUECHLE; PEÑA-LEGAZKUE, 2013; RANGA et al., 2016; VINIG; LIPS, 2015), creación de start-up (JONSSON; BARALDI; LARSSON, 2015), y servicios de incubación de empresas y parques tecnológicos (GONZÁLEZ-PERNÍA; KUECHLE; PEÑA-LEGAZKUE, 2013). En general, el objetivo último de la TT es comercializar la propiedad intelectual de la institución, ya sea para obtener ganancias financieras o una combinación entre logros económicos e impactos sociales (ALESSANDRINI; KLOSE; PEPPER, 2013).

Además de centrarse en el número de patentes registradas y los ingresos derivados de los acuerdos de licencia, la eficiencia de la función de TT se expresa en términos de parámetros como: el impacto social en las comunidades, la creación de nuevos empleos, la reducción de la probreza, un mayor desarrollo regional y un mayor crecimiento económico (ALESSANDRINI; KLOSE; PEPPER, 2013; RANGA et al., 2016); los cuales pueden ser equiparados en beneficios financieros a largo plazo para el país en su conjunto (ALESSANDRINI; KLOSE; PEPPER, 2013). El éxito de las activiades de TT se refleja en: 1) la creación de empleo; 2) el acceso a productos y servicios basados en tecnología; 3) la reducción de la probreza y la mortalidad; 4) la mejora en los indicadores de impacto social en las comunidades; y 5) los beneficios financieros a largo plazo para el país en su conjunto (ALESSANDRINI; KLOSE; PEPPER, 2013). Lo que el autor resalta es que la eficiente TT, impacta directamente a la sociedad de forma positiva; por tal motivo, en el conocimiento producido por la universidad deben estar involucrados los actores de la sociedad civil.

En ese sentido, la TT es de suma importancia para impulsar la vinculación de la universidad con su entorno (MARTÍNEZ et al., 2012); considerando como entorno a las empresas privadas, el Estado, las ONGs y la sociedad civil. Por lo tanto, cuando las universidades desarrollan actividades TT para vincularse con la sociedad, podrán o no recibir réditos económicos en función del actor que recibe el servicio. Es decir, las actividades de vinculación no necesariamente tiene un sentido de gratuidad; como lo propoponen las políticas públicas de educación superior de ciertos países de América Latina, como es el caso de Ecuador, que solo consideran como vinculación, actividades que se realizan sin fines de lucro a sectores vulnerables o de escasos recuros (CEAACES, 2014, 2015).

Esta interpretación parcial sobre el alcance de la vinculación con la sociedad puede ocasionar que las universidades: 1) subutilicen las capacidades de sus facultades y carreras; 2) no generen recursos para autofinanciar sus programas de TT y vinculación con la sociedad; 3) que se desarrollen programas y proyectos no alineados con las actividades de docencia e investigación de cada facultad; 4) que direccionen sus actividades para un único actor del entorno social. Sin embargo, para Martínez et al. (2012) la mayor oportunidad de vinculación con la colectividad que tienen las universidades latinoamericanas es mediante la prestación de servicios, implementación de programas de formación continua, y asistencia técnica en áreas 
de su competencia; posibilitando de esta forma la generación de recursos financieros, para autosustentar sus actividades.

Las universidades alcanzan un comportamiento socialmente responsable cuando la calidad y la cantidad de la investigación que efectúan, refuerza la relación entre universidad-entorno social, por medio del desarrollo de programas de transferencia de tecnología (MARTíNEZ et al., 2012). Precisamente, las facultades y el equipos de investigación son los responsables de generar las capacidades para absorver, crear y diseminar conocimiento (GONZÁLEZ-PERNÍA; KUECHLE; PEÑA-LEGAZKUE, 2013). Una implicación importante es que el conocimiento es intrínsecamente democrático por el hecho de que sus usuarios son en su mayoría externos al mundo académico - comunidades o sociedad civil (BOURKE, 2013). En consecuencia, tales actores están cada vez más involucrados en el co-diseño y co-producción del conocimiento, para de este modo harcerlo más relevante en el contexto de su aplicación y estrechamente relacionado con su eventual utilización en la sociedad (BOURKE, 2013).

Las universidades desempeñan un papel único en la sociedad, proporcionando un grupo de expertos en experimentación e innovación (NECOECHEA-MONDRAGÓN; PINEDA-DOMÍNGUEZ; SOTO-FLORES, 2013) y concibiendo la producción del conocimiento como un proceso socialmente distribuido (SCHIEFFER; LESSEM, 2014). Además, sus investigadores generan conocimiento el cual es aplicado en un contexto transdisciplinario, envolviendo una interacción cercana entre varios actores, que no son necesariamente académicos (SCHIEFFER; LESSEM, 2014). Por consiguiente, las universidades se vuelven una fuente de conocimiento externo para todos los actores sociales. Para que los países se beneficien de este conocimiento, los resultados de la investigación deben transferirse de la universidad a la sociedad; esta forma específica de valorización del conocimiento se conoce como transferencia de tecnología (VINIG; LIPS, 2015), que en consecuencia es vinculación con la sociedad. Sin embargo puede decirse que son pocos los casos en los que se ha establecido una colaboración de gran envergadura entre una universidad latinoamericana y el sector productivo y social (AROCENA; SUTZ, 2000).

En función de lo presentado en el primer apartado y en esta sección, la Fig. 2 presenta la relación entre TT y vinculación, según las actividades propias de cada dimensión y que las universidades deben desarrollar. Como se obserba en la figura, solo la participación en la vida social y cultural, y accción social son consideradas actividades puras de vinculación; el resto de actividades están relacionadas directamente con TT que tienen como insumo principal I+D que realiza la universidad.

Por lo tanto, las universidades tienen varias fuentes para realizar vinculación con la sociedad, la primera son los resultados de la investigación generada y la aplican por medio de transferencia de tecnología;

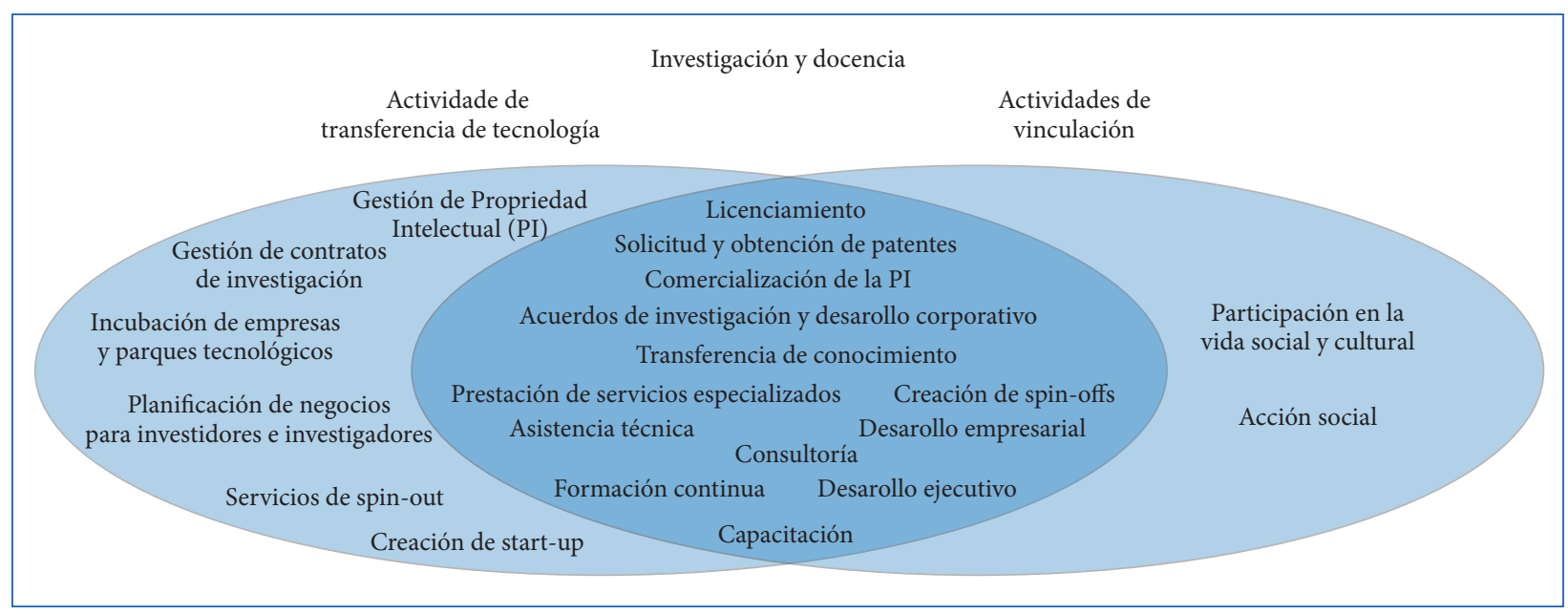

Figura 2. Relación entre TT y vinculación. 
la segunda en función de las capacidades que disponen, como por ejemplo cursos de extensión. Esta última no se basa en los resultados de la investigación sino en los recursos que tiene la institución (profesores, aulas, TICs, etc.). Las actividades de vinculación deben estar directamente relacionadas al grado de especialización de cada universidad ya que cada una debe responder a las necesidades externas a su manera, por lo que es vital que el apoyo del estado no conduzca a una contención directa o incluso a reducir su enfoque (HATAKENAKA, 2005). Por ejemplo, instituciones especializadas en ciencias duras, deben hacer actividades relacionadas a esos temas, de esa manera guarda una lógica la vinculación que están desarrollando. Si una institución realiza una actividad diferente de su esencia, tal actividad no será realizada de forma eficiente, ni tendrán una contribución de impacto de la universidad hacia la sociedad. Además, realizar vinculación con la sociedad no siempre implica el establecer proyectos de ese tipo, también pueden existir simplemente servicios de vinculación, como por ejemplo, consultorios odontológicos gratuitos o de bajo costo. Esto es una actividad de vinculación que no necesita ser un proyecto; como la institucionalidad lo exige en sus modelos de evaluación (CEAACES, 2015).

Por último, Valentín y Sánchez (2006) recomienda 6 acciones para superar los dificultades en la cooperación entre empresas y universidades: 1) marco legal que regule las relaciones entre ambas partes; 2) proteger la titularidad de las patentes que pudieran obtenerse por la I+D universitaria; 3 ) determinar los derechos de publicación de los resultados del investigador; 4) diseñar un adecuado sistema de incentivos y recompensas consistente con los objetivos de transferencia tecnológica; 5) identificar los factores que mejoran los niveles de éxito entre las partes; y, 6) figuras intermediarias entre la empresa y la universidad.

\section{Procedimientos metodológicos}

Para determinar si las prácticas de vinculación realizadas por las universidades son realmente de vinculación con la sociedad se analizó el universo de 60 universidades ecuatorianas públicas, privadas y cofinanciadas. Fue recolectada información de fuente secundaria disponible en el sitio web de cada institución; la información revisada corresponde a: 1) los reportes de rendición de cuentas; 2) los reglamentos y políticas asociados a la vinculación; 3) los reportes de vinculación; y, 4) los planes estratégicos vigentes (2016 y 2017). Al acceder a la página web de cada institución para descargar los documentos, simultáneamente se verificaba si la institución presentaba un departamento de vinculación formalmente establecido; en el caso de que dicha información no estuviera disponible en el sitio, se verificaba esa información en los documentos institucionales descargados. La información fue recolectada y analizada en un periodo de seis meses.

Los datos fueron analizados por medio de análisis de contenido con base en la revisión de la literatura presentada en la sección anterior, y en función de los siguientes constructos teóricos: a) La institución cuenta con un departamento formalmente establecido de vinculación con la sociedad; b) número y tipo de actividades vinculación que la institución ejecuta; c) coherencia de las actividades ejecutadas con el concepto de vinculación con la sociedad; d) relación de las actividades realizadas con transferencia de tecnología. Al analizar los datos según estos criterios los resultados mostrarán: la(s) actividad(es) de vinculación más aplicada por la institución; las actividades que sin serlas, las universidades las consideran como de vinculación con la sociedad; en qué criterios se fundamenta para considerar esas actividades como de vinculación; y las actividades de vinculación que también son de transferencia de tecnología.

Para identificar si las actividades ejecutadas por las universidades son de vinculación con la sociedad, la información fue contrastada con las principales actividades de vinculación y transferencia de tecnología establecidas en la literatura (Tabla 1). 
Tabla 1. Principales actividades de vinculación

\begin{tabular}{ccc}
\hline \multirow{2}{*}{ Actividad } & \multicolumn{2}{c}{ Tipo } \\
\cline { 2 - 3 } & Vinculación & Transferencia de tecnología \\
\hline Acción social & $\checkmark$ & \\
\hline Participación en la vida social y cultura & $\checkmark$ & $\checkmark$ \\
\hline Formación continua & $\checkmark$ & $\checkmark$ \\
\hline Servicios a la comunidad & $\checkmark$ & $\checkmark$ \\
\hline Capacitación & $\checkmark$ & $\checkmark$ \\
\hline Consultoría & $\checkmark$ & $\checkmark$ \\
\hline Prestación de servicios especializados & $\checkmark$ & $\checkmark$ \\
\hline Transferencia de tecnología & $\checkmark$ & $\checkmark$ \\
\hline Investigación-vinculación & $\checkmark$ & $\checkmark$ \\
\hline Creación de spin-offs (Emprendimiento) & $\checkmark$ & $\checkmark$ \\
\hline Asistencia técnica & $\checkmark$ & $\checkmark$ \\
\hline Solicitud y obtención de patentes & $\checkmark$ & $\checkmark$ \\
\hline Licenciamiento & $\checkmark$ & $\checkmark$ \\
\hline Comercialización de PI & $\checkmark$ & $\checkmark$ \\
\hline Acuerdos de I+D corporativo & $\checkmark$ & $\checkmark$ \\
\hline Desarrollo empresarial y ejecutivo & $\checkmark$ & $\checkmark$ \\
\hline
\end{tabular}

\section{Análisis y discusión}

En Ecuador, las Instituciones de Educación Superior (IES) están compuestas por Universidades y Escuelas Politécnicas. El país cuenta con 60 IES, 52 universidades y 8 escuelas politécnicas; de ese total el $55 \%$ son públicas, $32 \%$ privadas y $13 \%$ cofinanciadas (CES, 2018). Las dos provincias que más concentran IES en Ecuador son Pichincha y Guayas que en conjunto tienen el 52 \%. Estos datos muestran que: la educación superior en Ecuador es soportada por el Estado; y existe una concentración de universidades en las dos provincias principales del país.

Si bien todas estas instituciones realizan vinculación con la sociedad, los datos muestran que el $82 \%$ (49) tienen como parte de su estructura organizacional, un departamento que planifica organiza, dirige y controla la vinculación con la colectividad. Es decir, las actividades de vinculación en 8 de cada 10 universidades están formalmente institucionalizadas; esto demuestra que las universidades integran a la vinculación con la sociedad como una de sus actividades esenciales, y no la tratan como una actividad aislada o secundaria. Al analizar sus planes estratégicos para identificar si dentro de sus objetivos tenían alguno relacionado con la vinculación, se encontró que de las 60 instituciones, 52 tenían un plan estratégico actualizado, de estos, en 49 se establecía por lo menos un objetivo estratégico relacionado con vinculación con la sociedad. Esto corrobora el resultado anterior relacionado con la institucionalidad de la vinculación, las mismas 49 universidades con unidades formalmente establecidas establecen objetivos estratégicos de vinculación con la comunidad.

De esos objetivos, la mayoría sí se enmarcan dentro de la definición de vinculación con la sociedad establecida en la literatura; por ejemplo una universidad proponen como objetivo: 
- Desarrollar permanentemente programas de vinculación con la sociedad que permitan la difusión de los saberes y culturas, la prestación de servicios especializados, articulados a la docencia e investigación; para contribuir al desarrollo local y nacional.

Sin embargo, hay universidades que plantean objetivos parciales o distantes al concepto; por ejemplo:

- Disminuir los problemas sociales, mediante la ejecución de programas y proyectos de vinculación con la sociedad.

- Desarrollar la responsabilidad social en la comunidad universitaria.

De acuerdo a la información analizada, 20 son las actividades que las universidades consideran y desarrollan como acciones de vinculación con la sociedad. De ese total y de acuerdo a la literatura, ocho de ellas no son actividades de vinculación (Prácticas pre-profesionales y pasantías; formación de redes entre universidades; seguimiento a graduados; inserción laboral; movilidad estudiantil y docente; relaciones internacionales, actividades deportivas, y ayudantías de investigación). De las 12 restantes, dos son exclusivamente de vinculación (Acción social; y participación en la vida social y cultural) y 10 son actividades de vinculación y transferencia de tecnología simultáneamente (Formación continua; servicios a la comunidad; capacitación; consultoría; prestación de servicios especializados; transferencia de tecnología; investigación-vinculación; creación de spin-offs; asistencia técnica; y solicitud y obtención de patentes). Se puede observar que del total de actividades que ejecutan las universidades, solo el $60 \%$ son efectivamente de vinculación con la sociedad. Dicho de otra forma, de cada 10 acciones que realizan cuatro de ellas no son de vinculación con la sociedad; y sin embargo las instituciones creen que sí la son.

La Tabla 2 presenta cuantas universidades realizan cada una de las 20 actividades identificadas como de vinculación con la sociedad. Se observa que la acción social es la actividad más realizada por las universidades, ya que 55 (92 \%) la ejecutan. Esto se debe a que el CEAACES (2015) dentro de su Modelo de Evaluación solo evalúa esta actividad dentro de la vinculación con la colectividad, por lo que se evidencia que la misma institucionalidad estatal tiene una visión limitada de lo que conlleva la vinculación con la sociedad.

La Fig. 3 presenta proporcionalmente las actividades de vinculación realizadas por las universidades; las circunferencias de color más claro identifican las actividades que no son de vinculación, pero que las instituciones las consideran como tal. Los datos presentan una concentración en cuatro actividades: acción social, formación continua, servicios a la comunidad y prácticas pre-profesionales; no obstante, a esta última la literatura no la considera como de vinculación. Adicionalmente, se identificó que ninguna de las universidades desarrollan las siguientes actividades de vinculación con la sociedad: a) Licenciamiento, b) comercialización de PI, c) acuerdos de I+D corporativo, y d) desarrollo empresarial y ejecutivo.

Aunque se identificó que las universidades desarrollan 20 actividades, y ocho de las cuales no son de vinculación; no necesariamente cada una de las ellas ejecuta las 20 de forma simultánea. Según el número de actividades ejecutadas, las instituciones se pueden dividir en tres grupos: el primer grupo las universidades que realizan de una a seis actividades (30\%); el segundo grupo las que ejecuta entre siete a ocho actividades (34\%); y el tercer grupo de nueve hasta un total de 12 actividades (36\%) (Tabla 3 ).

Al comparar los reportes de rendición de cuentas con respecto a lo que cada institución declaraba como vinculación dentro de su reglamento; solo tres universidades coincidían entre lo que declaraban en su informe de rendición con lo establecido en su reglamento. El 56 \% de las instituciones tenían más del $50 \%$ 
Tabla 2. Actividades de vinculación declaradas por las Universidades

\begin{tabular}{|c|c|c|c|c|c|}
\hline Actividad & Vinculación & $\begin{array}{l}\text { \# Universidades que } \\
\text { realiza actividad }\end{array}$ & $\begin{array}{l}\text { Respecto al total } \\
\text { universidades }\end{array}$ & $\begin{array}{l}\text { Respecto total } \\
\text { actividades }^{\star \star}\end{array}$ & Acumulado \\
\hline Acción social (AS) & Sí & 55 & $92 \%$ & $12 \%$ & $12 \%$ \\
\hline $\begin{array}{l}\text { Formación continua } \\
\text { (FC) }\end{array}$ & Sí & 42 & $70 \%$ & $9 \%$ & $21 \%$ \\
\hline $\begin{array}{l}\text { Servicios a la } \\
\text { comunidad (SC) }\end{array}$ & Sí & 42 & $70 \%$ & $9 \%$ & $31 \%$ \\
\hline $\begin{array}{l}\text { Prácticas pre- } \\
\text { profesionales y } \\
\text { pasantías (PPP) }\end{array}$ & No & 38 & $63 \%$ & $8 \%$ & $39 \%$ \\
\hline $\begin{array}{c}\text { Participación en la } \\
\text { vida social y cultural } \\
\text { (VSC) }\end{array}$ & Sí & 31 & $52 \%$ & $7 \%$ & $46 \%$ \\
\hline Capacitación (Ca) & Sí & 30 & $50 \%$ & $7 \%$ & $53 \%$ \\
\hline Consultoría (Co) & Sí & 28 & $47 \%$ & $6 \%$ & $59 \%$ \\
\hline $\begin{array}{l}\text { Prestación de servicios } \\
\text { especializados (SE) }\end{array}$ & Sí & 27 & $45 \%$ & $6 \%$ & $65 \%$ \\
\hline $\begin{array}{l}\text { Redes universitarias } \\
\text { (R) }\end{array}$ & No & 26 & $43 \%$ & $6 \%$ & $71 \%$ \\
\hline $\begin{array}{l}\text { Seguimiento a } \\
\text { graduados (SG) }\end{array}$ & No & 24 & $40 \%$ & $5 \%$ & $76 \%$ \\
\hline $\begin{array}{l}\text { Investigación- } \\
\text { vinculación (IV) }\end{array}$ & Sí & 19 & $32 \%$ & $4 \%$ & $80 \%$ \\
\hline $\begin{array}{l}\text { Transferencia de } \\
\text { tecnología (TT) }\end{array}$ & Sí & 18 & $30 \%$ & $4 \%$ & $84 \%$ \\
\hline Inserción laboral (IL) & No & 16 & $27 \%$ & $4 \%$ & $88 \%$ \\
\hline $\begin{array}{c}\text { Creación de spin-offs } \\
\text { (Emprendimiento) } \\
\text { (CSF) }\end{array}$ & Sí & 14 & $23 \%$ & $3 \%$ & $91 \%$ \\
\hline $\begin{array}{l}\text { Movilidad estudiantil } \\
\text { y docente (MED) }\end{array}$ & No & 14 & $23 \%$ & $3 \%$ & $94 \%$ \\
\hline $\begin{array}{c}\text { Relaciones } \\
\text { internacionales (RI) }\end{array}$ & No & 11 & $18 \%$ & $2 \%$ & $96 \%$ \\
\hline $\begin{array}{l}\text { Asistencia técnica } \\
\text { (AT) }\end{array}$ & Sí & 8 & $13 \%$ & $2 \%$ & $98 \%$ \\
\hline Deportes (D) & No & 6 & $10 \%$ & $1 \%$ & $99 \%$ \\
\hline $\begin{array}{c}\text { Ayudantías de } \\
\text { investigación (AI) }\end{array}$ & No & 2 & $3 \%$ & $0 \%$ & $100 \%$ \\
\hline $\begin{array}{l}\text { Solicitud y obtención } \\
\text { de patentes (SOP) }\end{array}$ & Sí & 1 & $2 \%$ & $0 \%$ & $100 \%$ \\
\hline
\end{tabular}




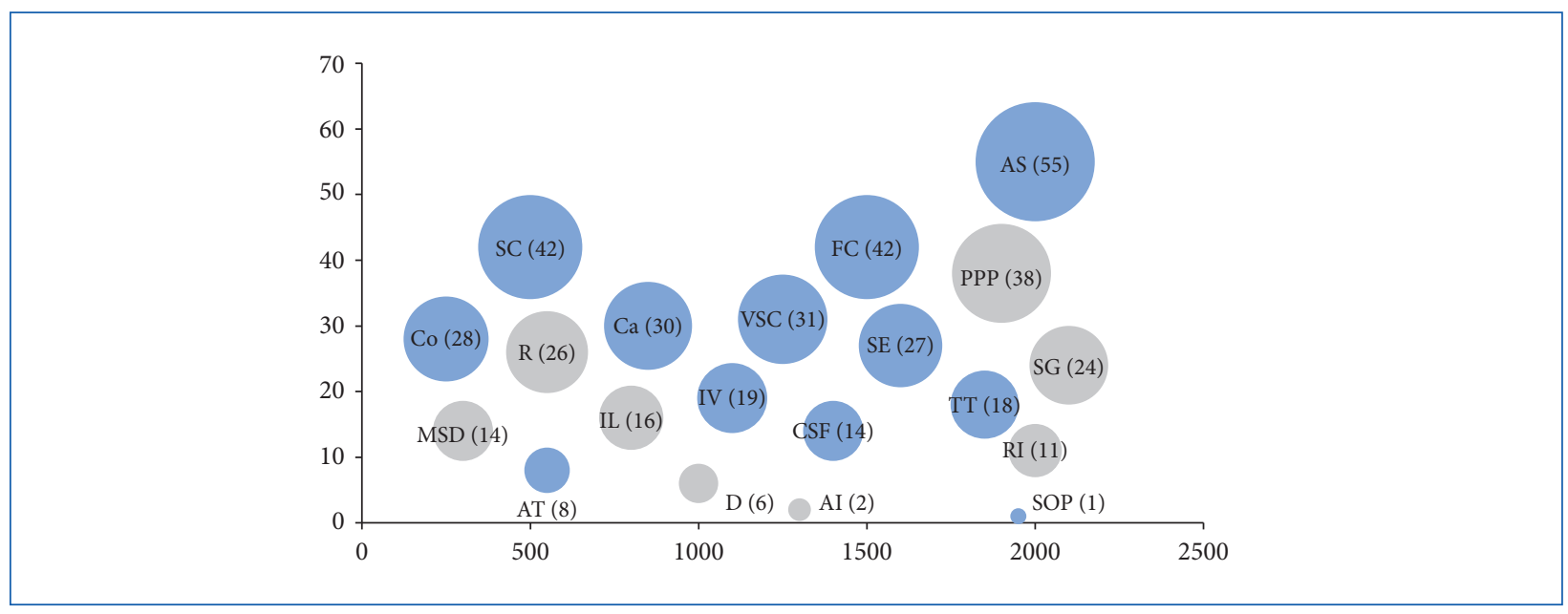

Figura 3. Actividades de vinculación realizadas.

Tabla 3. Total de actividades de vinculación con la sociedad declaradas.

\begin{tabular}{|c|c|c|c|c|}
\hline Grupo & $\begin{array}{l}\text { \# Actividades } \\
\text { declaradas }\end{array}$ & \# Universidades & Porcentaje & $\begin{array}{c}\text { Porcentaje acumulado } \\
\text { por grupo }\end{array}$ \\
\hline \multirow{5}{*}{1} & 1 & 5 & $8 \%$ & \\
\hline & 2 & 1 & $2 \%$ & \\
\hline & 3 & 3 & $5 \%$ & \\
\hline & 5 & 5 & $8 \%$ & \\
\hline & 6 & 4 & $7 \%$ & $30 \%$ \\
\hline \multirow{2}{*}{2} & 7 & 10 & $17 \%$ & \\
\hline & 8 & 10 & $17 \%$ & $34 \%$ \\
\hline \multirow{4}{*}{3} & 9 & 9 & $15 \%$ & \\
\hline & 10 & 6 & $10 \%$ & \\
\hline & 11 & 3 & $4 \%$ & \\
\hline & 12 & 4 & $7 \%$ & $36 \%$ \\
\hline \multicolumn{2}{|c|}{ Total } & 60 & $100 \%$ & \\
\hline
\end{tabular}

de discrepancia entre lo que tienen como actividades de vinculación dentro de su reglamento frente a lo que reportan en su informe de rendición de cuentas. Esto refleja que las universidades no planifican, no ejecutan, ni evalúan sus actividades en función de la visión de futuro que tienen (Tabla 4).

Como parte de esa discrepancia se identificó que las universidades en un 7 \% tienen actividades de vinculación que reportan fuera de esta dimensión, aunque la consideraban parte. De la misma forma, se detectó que las instituciones en un 18 \% reportaban actividades dentro de vinculación, aunque en sus reglamentos no las incluían (Tabla 5).

\section{Conclusiones}


Tabla 4. Porcentaje de actividades de vinculación que coinciden con el informe de rendición de cuentas

\begin{tabular}{cccc}
\hline & \% de AV & Frecuencia & \% Acumulado \\
\hline $0 \%$ & $10 \%$ & 2 & 4 \\
\hline $10 \%$ & $20 \%$ & 7 & 16 \\
\hline $20 \%$ & $30 \%$ & 7 & 48 \\
\hline $30 \%$ & $40 \%$ & 9 & 56 \\
\hline $40 \%$ & $50 \%$ & 7 & 75 \\
\hline $50 \%$ & $60 \%$ & 11 & 88 \\
\hline $60 \%$ & $70 \%$ & 7 & 95 \\
\hline $70 \%$ & $80 \%$ & 4 & 95 \\
\hline $801 \%$ & $90 \%$ & 0 & 100 \\
\hline $90 \%$ & $100 \%$ & 3 & \\
\hline
\end{tabular}

${ }^{\star}$ De las 60 universidades se obtuvo el informe de 57

Tabla 5. Reporte de actividades de vinculación

\begin{tabular}{ccc}
\hline Estado & Total actividades & Porcentaje \\
\hline Si reporta & 207 & 47 \\
\hline No reporta & 122 & 28 \\
\hline Reporta actividades fuera de vinculación, aunque las consideraban dentro & 33 & 7 \\
\hline Reporta actividades dentro de vinculación, aunque las consideraba fuera & 80 & 18 \\
\hline Total & 442 & 100 \\
\hline
\end{tabular}

El objetivo de esta investigación fue determinar si las prácticas de vinculación realizadas por las universidades son realmente de vinculación con la sociedad, para lo cual se realizó un estudio a partir de los planes estratégicos, reglamentos y reportes de rendición de cuentas de cada una, comparando sus declaraciones de actividades de vinculación con lo enunciado por la literatura pertinente. Se observa que en Ecuador, las universidades conciben parcialmente a la vinculación con la sociedad de acuerdo a los parámetros mundialmente aceptados, por lo tanto, las actividades que ejecutan no siempre corresponden a esta misión de las universidades; como consecuencia la vinculación no se mide como se debe medir. Por ejemplo, la actividad de seguimiento a graduados que debería servir como insumo para hacer ajustes en las redes curriculares de las carreras, se considera como actividad de vinculación con la sociedad, en esta misma línea se ha podido detectar que las actividades deportivas se declaran dentro de vinculación. Por otro lado, las IES ejecutan actividades de formación continua cuya naturaleza es precisamente servir de enlace entre la universidad y la sociedad, sin embargo, no se la reconoce como tal por parte de las mismas instituciones. Es decir, existe un conocimiento parcial sobre lo que implican las actividades de vinculación con la sociedad.

Uno de los puntales de la vinculación es la transferencia de tecnología obtenida como producto de actividades de investigación; sin embargo, esta actividad tiene poco desarrollo dentro de las universidades, la razón es que la vinculación se enfoca en el sector social dejando a un lado la industria lo cual hace que exista un divorcio entre la academia y el aparato productivo, lazo que en otras sociedades permite un 
avance acelerado de la economía. Finalmente, existe la visión dentro de las universidades ecuatorianas y latinoamericanas -sean estas públicas, privadas o cofinanciadas- que el papel de la universidad con respecto a la vinculación es encargarse de resolver los problemas que el Estado y sus instituciones no puede resolver en temas de salud, pobreza y desempleo.

\section{Contribución de los autores}

Conceptualización de la investigación, Acosta B; Revisión de la literatura, Rueda I, Acosta B; Instrumentos de recolección y análisis de dados: Rueda I, Acosta B; Análisis de resultados, Rueda I, Acosta B, Cueva F; Discusión; Rueda I, Acosta B, Cueva F; Escritura, Rueda I, Acosta B; Corrección de estilo, Cueva F.

\section{Referencias}

ALESSANDRINI, M.; KLOSE, K.; PEPPER, M. S. University entrepreneurship in South Africa: Developments in technology transfer practices. Innovation: Organization \& Management, Reino Unido, v. 15, n. 2, p. 205 214, 2013. https://doi.org/10.5172/impp.2013.15.2.205.

AROCENA, R.; SUTZ, J. La Universidad Latinoamericana del Futuro. México: Colección UDUAL, 2000.

AUDRETSCH, D. B. From the entrepreneurial university to the university for the entrepreneurial society. Journal of Technology Transfer, Estados Unidos, v. 39, n. 3, p. 313-321, 2014. https://doi.org/10.1007/ s10961-012-9288-1.

BEZERRA, R. A relação universidade-sociedade na periferia do capitalismo. Revista Brasileira de Ciências Sociais, São Paulo, v. 27, n. 78, p. 25-40, 2012. https://doi.org/10.1590/S0102-69092012000100002.

BOURKE, A. Universities, Civil Society and the Global Agenda of Community-engaged Research. Globalisation, Societies and Education, Reino Unido, v. 11, n. 4, p. 498-519, 2013. https://doi.org/10.1080 /14767724.2013.834182.

CEAACES. Resolución No. 104-CEAACES-SO-12-2014 - Reglamento de Evaluación, Acreditación y Categorización de Carreras de las Instituciones de Educación Superor. Ecuador: CEAACES, 2014.

CEAACES. Modelo de Evaluación Institucional de Universidades y Escuelas Politécnicas. Ecuador: CEAACES, 2015.

CES. Instituciones de Educación Superior. Ecuador: CES, 2018. Disponible en: http://www.ces.gob.ec/ index.php?option=com_sobipro\&sid=68:Instituciones-de-Educacion-Superior\&Itemid=0. Acceso en: 25 de abril de 2018.

FENG, H.; CHEN, C.; WANG, C. The role of intellectual capital and university technology transfer offices in university-based technology transfer. The Service Industries Journal, Reino Unido, v. 32, n. 6, p. 899-917, 2012. https://doi.org/10.1080/02642069.2010.545883.

GONZÁLEZ-PERNÍA, J. L.; KUECHLE, G.; PEÑA-LEGAZKUE, I. An Assessment of the Determinants of 
University Technology Transfer. Economic Development Quarterly, Kalamazoo, v. 27, n. 1, p. 6-17, 2013. https://doi.org/10.1177/0891242412471847.

HATAKENAKA, S. Development of third stream activity: Lessons from international experience. Oxford: Higher Education Policy Institute, p. 1-35, 2005.

JONSSON, L.; BARALDI, E.; LARSSON, L.-E. A broadened innovation support for mutual benefits: Academic engagement by universities as part of technology transfer. International Journal of Technology Management \& Sustainable Development, Bristol, v. 14, n. 2, p. 71-91, 2015. https://doi.org/10.1386/tmsd.14.2.71_1.

MARTÍNEZ, C. D. M.; MAVÁREZ, L. R.; ROMERO, J. G.; ANTÚNEZ, N. Responsabilidad social universitaria, transferencia y mercadeo tecnológico: vinculación con el entorno social. Revista Venezolana de Gerencia, Venezuela, v. 17, n. 59, p. 512-528, 2012. https://doi.org/10.31876/revista.v17i59.10912.

MARTÍNEZ, M. R. V.; SOTO, A. Modalidades de transferencia tecnológica en la vinculación universidadsector productivo: Motivaciones y obstáculos. Revista de Ciencias Sociales, San Pedro, v. 13, n. 2, p. 290 302, 2007. Disponible en: https://dialnet.unirioja.es/servlet/articulo? codigo=4247091. Acceso en: 14 de marzo de 2018.

NECOECHEA-MONDRAGÓN, H.; PINEDA-DOMÍNGUEZ, D.; SOTO-FLORES, R. A conceptual model of technology transfer for public universities in Mexico. Journal of Technology Management and Innovation, Santiago, v. 8, n. 4, p. 24-35, 2013. https://doi.org/10.4067/S0718-27242013000500003.

PALEARI, S.; DONINA, D.; MEOLI, M. The role of the university in twenty-first century European society. Journal of Technology Transfer, Estados Unidos, v. 40, n. 3, p. 369-379, 2015. https://doi.org/10.1007/ s10961-014-9348-9.

RANGA, M; TEMEL, S; AR, I. M.; YESILAY, R. B.; SUKAN, F. V. Building Technology Transfer Capacity in Turkish Universities: A critical analysis. European Journal of Education, Hoboken, v. 51, n. 1, p. 90-106, 2016. https://doi.org/10.1111/ejed.12164.

SCHIEFFER, A.; LESSEM, R. The Integral University: Holistic development of individuals, communities, organisations and societies. Prospects: Comparative Journal of Curriculum, Learning, and Assessment, Inglaterra, v. 44, n. 4, p. 607-626, 2014. https://doi.org/10.1007/s11125-014-9324-z.

SCHOEN, A.; POTTERIE, B. P.; HENKEL, J. Governance typology of universities' technology transfer processes. Journal of Technology Transfer, Estados Unidos, v. 39, n. 3, p. 435-453, 2014. https://doi. org/10.1007/s10961-012-9289-0.

SOLEIMANI, M.; TABRIZ, A. A.; SHAVARINI, S. K. Developing a Model to Explain the Process of Technology Transfer at Entrepreneurial University. Industrial Engineering and Management Systems, Seul, v. 15, n. 4, p. 298-306, 2016. https://doi.org/10.7232/iems.2016.15.4.298.

TSENG, A. A.; RAUDENSKY, M. Performance Evaluations of Technology Transfer Offices of Major US Research Universities in 2012/2013. Journal of Technology Management \& Innovation, Singapur, v. 9, n. 1, p. 93-102, 2014. https://doi.org/10.4067/S0718-27242014000100008.

VALENTÍN. E. M. M.; SÁNCHEZ, M. A. M. Hacia una gestión eficaz de las relaciones entre empresas y universidades. Universia Business Review, Madrid, n. 10, p. 38-53, 2006. Disponible en: https://dialnet. 
unirioja.es/servlet/articulo?codigo=1993926. Acceso en: 20 de agosto de 2018 .

VINIG, T.; LIPS, D. Measuring the performance of university technology transfer using meta data approach: the case of Dutch universities. Journal of Technology Transfer, Estados Unidos, v. 40, n. 6, p. 1034-1049, 2015. https://doi.org/10.1007/s10961-014-9389-0.

\section{Sobre los autores}

Iván Rueda es profesor de la Pontificia Universidad Católica del Ecuador (PUCE), Facultad de Ciencias Administrativas, Quito (Pichincha), Ecuador. Pregrado en Administración de Empresas y candidato a doctor en Administración de empresas por la Universidad Nacional de Rosario. Docente-investigador en desempeño organizacional en instituciones de educación superior.

Byron Acosta es profesor de la Escuela Politécnica Nacional (EPN), en el Programa de doctorado en Gestión Tecnológica, Departamento de ciencias administrativas, Quito (Pichincha), Ecuador. Pregrado en Administración y Doctorado en Innovación y Desarrollo Sustentable por la Universidade Federal do Río Grande do Sul. Docente-investigador en gestión de la tecnología y la producción.

Fabián Cueva es profesor de la Pontificia Universidad Católica del Ecuador (PUCE), Facultad de Ciencias Administrativas, Quito (Pichincha), Ecuador. Pregrado en Administración de Empresas y candidato a doctor en Administración de empresas por la Universidad Nacional de Rosario. Docente-investigador en capacidad de absorción en organizaciones deportivas.

Recibido: 28 Dic 2018

Acepto: 04 Dic 2019 